\title{
Short Paper: Frequency Regulation Services from Connected Residential Devices
}

\author{
Kyri Baker ${ }^{\star}$, Xin Jin, Deepthi Vaidhynathan, Wesley Jones, Dane Christensen, \\ Bethany Sparn, Jason Woods, Harry Sorensen, and Monte Lunacek \\ National Renewable Energy Laboratory \\ Golden, CO, USA \\ *Corresponding author: kyri.baker@nrel.gov
}

\begin{abstract}
This paper demonstrates potential benefits that residential buildings can provide for frequency regulation services in the electric power grid. In a hardware-in-the-loop (HIL) implementation, simulated homes and a physical laboratory home are coordinated via a grid aggregator, and it is shown that their aggregate response has the potential to follow the regulation signal on a timescale of seconds. Connected (communication-enabled) devices in the National Renewable Energy Laboratory's (NREL's) Energy Systems Integration Facility (ESIF) received demand response (DR) requests from a grid aggregator, and the devices responded to meet the signal while satisfying comfort bounds and physical hardware limitations. Future research will address the issues of cybersecurity threats, participation rates, and reducing equipment wear-and-tear while providing grid services.
\end{abstract}

\section{CCS Concepts}

-Computing methodologies $\rightarrow$ Modeling and simulation; Agent / discrete models;

\section{Keywords}

Frequency regulation; demand response; smart appliances; building-to-grid; model predictive control

\section{INTRODUCTION}

With the increase in so-called "smart" devices in residential homes comes an increased opportunity for these homes to provide services to the electric power grid through improvements in control and communication. Currently, the majority of these services amount to responding to price signals or participating in direct load control; however, in this project we explore the capabilities of residential buildings to provide frequency regulation grid services. Frequency regulation is a service, usually on the timescale of every 4 seconds, provided by both generation and load to maintain the system frequency at its nominal value $-60 \mathrm{~Hz}$ in the United

ACM acknowledges that this contribution was authored or co-authored by an employee, or contractor of the national government. As such, the Government retains a nonexclusive, royalty-free right to publish or reproduce this article, or to allow others to do so, for Government purposes only. Permission to make digital or hard copies for personal or classroom use is granted. Copies must bear this notice and the full citation on the first page. Copyrights for components of this work owned by others than ACM must be honored. To copy otherwise, distribute, republish, or post, requires prior specific permission and/or a fee. Request permissions from permissions@acm.org.

BuildSys '16, November 16-17, 2016, Palo Alto, CA, USA

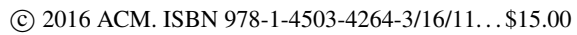

DOI: http://dx.doi.org/10.1145/2993422.2993569
States, for example - by maintaining the balance between supply and demand [1]. When supply exceeds demand, the frequency in the grid increases. When demand exceeds supply, the frequency in the grid decreases.

Traditionally, the majority of frequency regulation in the grid has been provided by traditional large-scale generators; however, as more inverter-interfaced distributed generation (such as rooftop photovoltaics) enters the power system, it becomes increasingly important to develop control methods and technologies that are capable of maintaining this balance. In this paper, we explore the possibilities for large populations of connected residential loads to participate in maintaining this balance by adjusting their power consumption with automatic control of connected home devices.

Extensive research has been performed with regard to the capabilities of electric vehicles (EVs) to provide frequency regulation $[2,3]$ as well as commercial buildings to provide grid services $[4,5]$, including control of aggregate commercial buildings [6]. Recently, residential buildings have been garnering attention in the area of fast-timescale grid services, specifically in the area of thermostatically controlled loads (TCL) [7]. However, much of this research focused on economic analysis and simulation, rather than demonstrating these potentials with physical hardware. In [8], physical household refrigerators representing TCLs were controlled in response to a demand response request from an aggregator; however, the DR event is on a slower timescale (hours).

In this paper, we consider a 10 -second level DR request, representing the percentage of load an individual household should reduce or increase. As expected, each individual home will not be able to follow a signal on this timescale with full accuracy, which is why an aggregator is modeled. It is shown that in aggregate, many homes participating in this service are able to follow a frequency regulation request; however, inaccurate equipment models, communication and hardware delays, and equipment safety and longevity concerns may prevent these appliances from being the optimal choice for fast-timescale frequency regulation.

One physical home located in the ESIF is tested with a number of simulated homes to fulfill this request. In the physical home, a HIL setup is used to represent the building thermal characteristics and operate the air conditioner (AC) under user-specified weather conditions, which could be from any climate zone across the United States. A suite of home appliances follow the request with as much accuracy as possible while fulfilling hardware constraints such as minimum on/off times and user constraints such as air and water temperature, as well as EV charging level requirements. Re- 


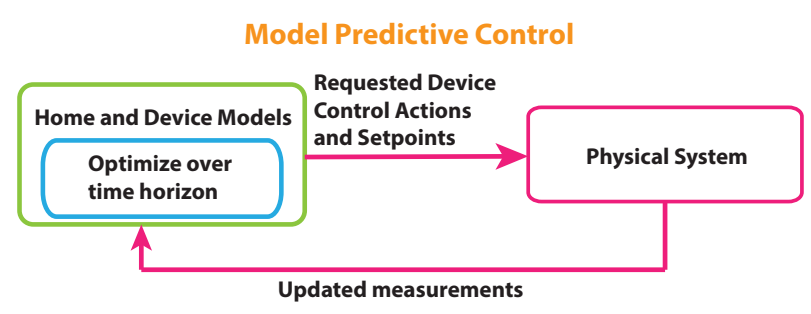

Figure 1: Diagram depicting model predictive control.

sults from the laboratory experiment are shown as well as results in conjunction with simulated households to demonstrate the benefit of residential buildings participating in fast-timescale grid services while satisfying user preferences. Issues regarding the implementation of this fast-timescale appliance control are discussed, as well as directions for potential future work in this area.

\section{HARDWARE LAYOUT}

The considered connected devices in ESIF for the frequency regulation experiment include an electric vehicle supply equipment (EVSE) unit and a Wi-Fi-enabled thermostat, which interfaces with a residential AC unit. VOLTTRON [9] as an agent-based control platform simulates the aggregator, which receives the estimated demand response capacity of each home and determines the frequency regulation request to each individual home.

The EVSE considered in the demonstration has three potential charging statuses: off (50 Watt standby consumption), curtailed-rate $(1.7 \mathrm{~kW})$, and full-rate $(4.1 \mathrm{~kW})$. The EVSE communicates with an EV using the SAE J1772 protocol, which is a North America standard for EV electrical connectors. The EVSE communicates with the VOLTTRON system using the CTA-2045 standard, which is a modular connection interface for demand response.

The AC is connected to a Wi-Fi enabled thermostat, which is controlled via the application programming interface (API) provided by the manufacturer. The $\mathrm{AC}$ unit consumes about $1.2 \mathrm{~kW}$ when operating; however, the actual power is dependent on the ambient conditions. Simulated outdoor temperature around the condenser is controlled with an environmental chamber to match a weather file. The indoor conditions are determined based on an EnergyPlus building model running in real time and are used to control the temperature around the thermostat and the return air for the air handler.

\section{CONTROL LOGIC AND ARCHITECTURE}

\subsection{Model Predictive Control}

Model predictive control (MPC), or receding horizon control, is a technique that calculates control decisions for the current timestep while taking into account the future state of the system, calculated from a model of the actual system [10]. As it can be seen in Figure 1, models of the home and connected appliances are formed and control decisions are made over the time horizon considering these models. Here, these control decisions aim to minimize the difference between requested and actual regulation provided while keeping user comfort requirements as constraints. Then, the requested actions are sent to physical devices, and their response is measured and sent back to the controller.

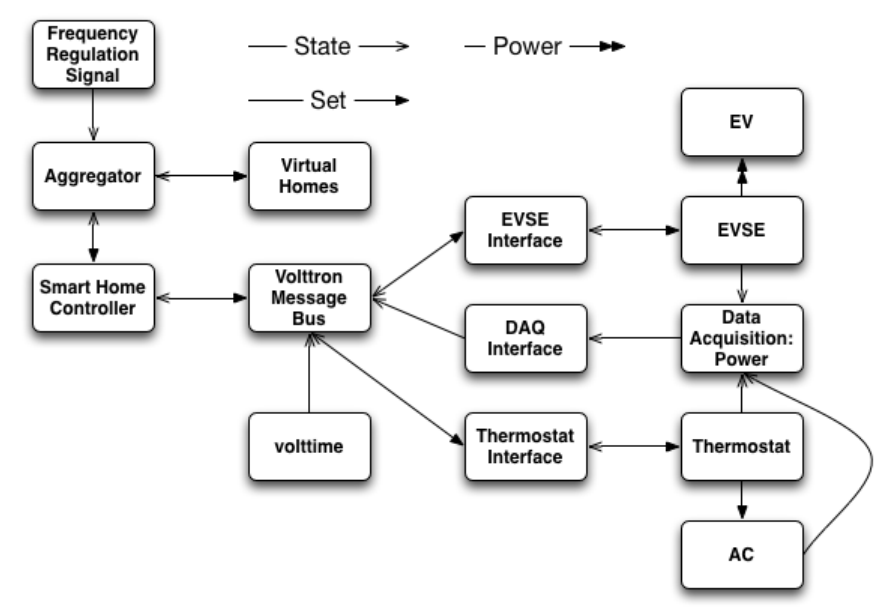

Figure 2: Hardware-in-the-loop architecture.

\subsection{Architecture}

The agent-based platform VOLTTRON was used for HIL simulations. The aggregator was modeled as an agent and simulates virtual smart homes/devices that can participate in frequency regulation. The aggregator receives a frequency regulation signal from the utility every 10 seconds. The aggregator queries the HIL home for its available regulation capacity and calculates a portion of the total regulation for the HIL home, depending on the home's current DR capabilities. It then dispatches the frequency regulation requests to the simulated homes.

The smart home controller then uses this assigned capacity to formulate a control strategy to provide the closest possible level of frequency regulation to the requested level without compromising user comfort. The appliances are controlled through an interface agent that serves as a wrapper around the appliances' communication API. The response of the appliances are measured by their power consumption through a data acquisition system. Figure 2 shows the setup used for the HIL experiment. The volttime agent is a simulation time manager in the VOLTTRON environment, publishing a timestamp that all interfaces and controllers use to synchronize their execution. Volttime enables running the experiments in real time, drawing data from a particular day and time.

\section{FREQUENCY REGULATION}

In the following diagrams, an actual regulation signal from PJM Interconnection in July 2013, sampled every 10 seconds, is used as the input to the aggregator agent.

\subsection{Actual Device Response}

In the smart home controller, simplified linear models were formed for the EV and AC (physical home) and $\mathrm{AC}$ and heat pump water heaters (HPWH) (simulated homes). Figure 3, shows the graphical user interface used to enable/disable the inclusion of physical hardware into the frequency regulation algorithm. The aggregator agent determines what load shed/add request to send each home considered in the demonstration, and the response of the house agent is recorded and displayed every 10 seconds. In the user interface depicted in Figure 3, the response from the 


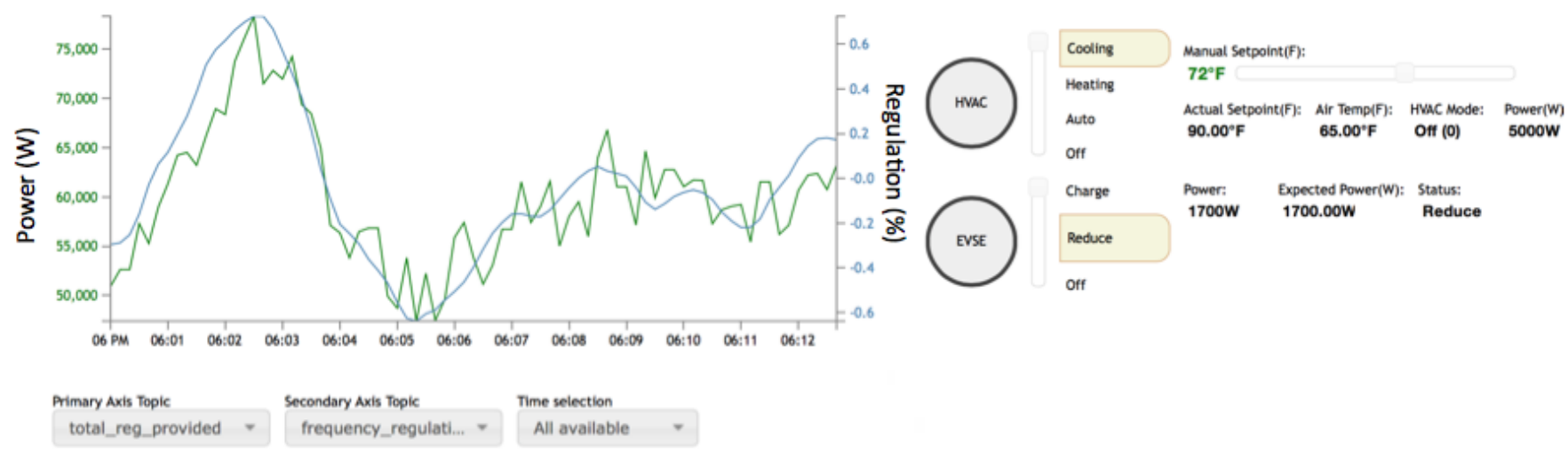

Figure 3: Graphical user interface for connected devices.

laboratory home is plotted against the requested response from the aggregator.

As seen in Figure 4, the actual combined EV and AC response from the physical home does not always match the request calculated from the model. Some complex dynamics of the $\mathrm{AC}$ are not captured, including the inrush current that briefly occurs at startup. In addition, the EVSE has a delay when the charging rate is modified; this is both a factor of physical limitations of the relay as well as a safety feature of the equipment. This delay can be seen when examining the plot: the response of the laboratory home slightly lags the response of the model. In the linear model for the home, a lumped capacitance model was considered, where model parameters were fit to a more detailed EnergyPlus model.

Despite the inconsistencies between the hardware and model responses, the linear models for the appliances and home are used in the simulations for multiple reasons. First, home energy management systems (HEMS) in the future will likely be performing computations on an embedded platform, potentially on a seconds timescale. Thus, having complex models renders the optimization problem computationally burdensome. Second, when deployed in an arbitrary home, the controller would not have full knowledge of the details of each connected device or of the home itself, so making more detailed assumptions about the actual devices may result in suboptimal performance of the controller.

Figure 5 depicts the simulated indoor air temperature with and without providing regulation services. The upper and lower bounds on the deadband are $24^{\circ} \mathrm{C}$ and $26^{\circ} \mathrm{C}$, respectively. These bounds are still satisfied by the home energy management controller's decisions; however, when providing response to the regulation request, the $\mathrm{AC}$ cycles more often. The physical limitations for on/off time from the hardware were included in the simulation (for example, the AC must stay off for at least 5 minutes to avoid damaging the equipment); however, long-term lifetime impacts on the appliance operation were not considered here.

Figure 6 shows the evolution of the actual home temperature over time as the $\mathrm{AC}$ cycles to meet frequency regulation requests. As seen in the figure, the temperature of the home is kept within the user-specified boundaries while providing grid services. To avoid the congestion on the message bus, values were only published every second, which is why the $\mathrm{AC}$ spike on the sub-second level sometimes does not appear in the figure.

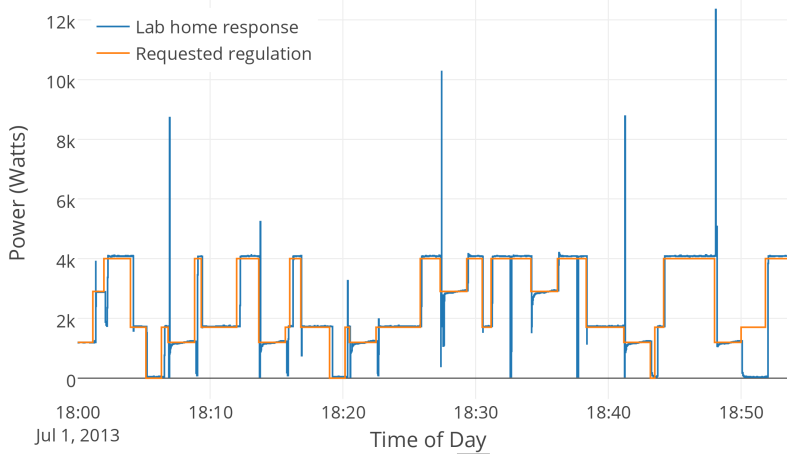

Figure 4: Requested device response versus actual device response.

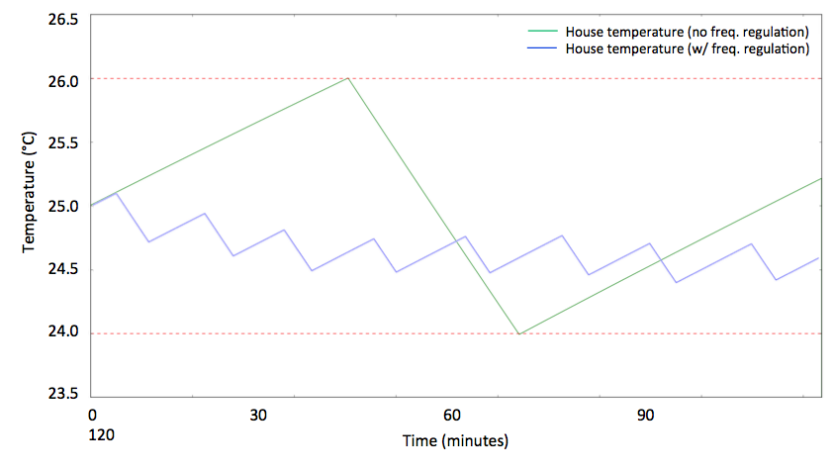

Figure 5: Simulated home temperature with and without providing frequency regulation services.

\subsection{Aggregation of Simulated Homes}

As the devices in the laboratory home were being controlled, many loads from simulated houses were controlled to match the actual frequency regulation signal, as seen in Figure 7 . The simulated homes, with appliances represented by linear models, were randomly populated with HPWH and $\mathrm{AC}$ devices of a specified power consumption. The laboratory home devices plus all the devices in the simulated homes are controlled so that their cumulative power follows the frequency regulation signal as closely as possible. The simulated devices are shown at the bottom of the figure; roughly a $1003-5 \mathrm{~kW}$ on/off loads are considered, but no 


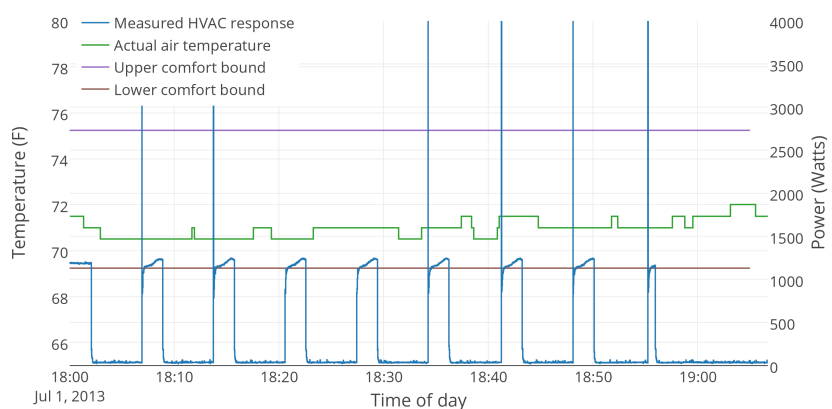

Figure 6: Air conditioner power consumption and indoor air temperature in HIL home while providing frequency regulation services.

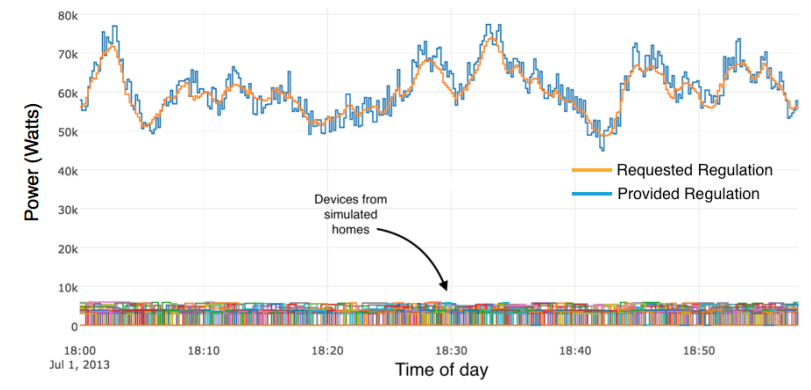

Figure 7: Aggregate simulated devices plus the laboratory home providing 10-second grid services.

more than 20 or 30 are on simultaneously. In aggregate, even though the devices do not have a continuous, controllable range of power consumptions, the response from the aggregate homes is able to follow the regulation signal more smoothly than a smaller number of homes due to the law of large numbers.

However, in practice, the models used in the simulations will not be exact representations of how the appliances would respond in reality. The complex dynamics of AC systems and water heaters may not be suitable to model in a realtime HEMS application; for example, capturing the power spikes in Figures 4 and 6 would most likely require higherorder models. Thus, the aggregation of these devices could potentially help smooth out the inconsistencies with the models used to determine the controller actions.

\section{CONCLUSION AND LESSONS LEARNED}

This paper presented insights into the potential for aggregated residential buildings to provide frequency regulation services. In addition to a physical hardware demonstration using the VOLTTRON platform, the results from larger numbers of aggregated homes are shown to demonstrate the effect of many connected devices responding in aggregate. Even with simplified linear models of the considered appliances, when considered in aggregate, residential buildings can prove to be beneficial for providing fast-timescale grid services. The results are encouraging for future work in this space, especially with the increasing penetration of controllable, connected devices at the home level.

Further research would be beneficial in analyzing how connected devices could participate on this timescale without greatly impacting their lifetime. Because of this, and partially due to model inaccuracies and equipment limitations, participation in frequency regulation at the residential level may be more beneficial on slightly longer timescales (i.e., minutes). Aggregation will certainly help in this respect. In addition, the results presented in this paper assumed that all of the devices were responsive, that many of their parameters and physical safety and hardware limitations were already known, and that the aggregator and homes were able to have fast, bi-directional communication between each other. Future work will address potential cybersecurity threats, how to learn system parameters over time with limited information (perhaps using a system identification approach), and how to better ensure the lifetime and wear-and-tear of the equipment is not exacerbated by the control scheme.

\section{REFERENCES}

[1] B. Kirby, "Spinning reserve from responsive loads," Oak Ridge National Laboratory Technical Report ORNL/TM-2003/19, March 2003.

[2] J. Donadee and M. Ilic, "Stochastic optimization of grid to vehicle frequency regulation capacity bids," IEEE Trans. on Smart Grid, vol. 5, no. 2, pp. 1061-1069, Mar 2014.

[3] W. Kempton, V. Udo, K. Huber, K. Komara, S. Letendre, S. Baker, D. Brunner, and N. Pearre, "A test of vehicle-to-grid (v2g) for energy storage and frequency regulation in the PJM system," in Mid-Atlantic Grid Interactive Cars Consortium, 2009.

[4] M. Maasoumy, B. M. Sanandaji, A. Sangiovanni-Vincentelli, and K. Poolla, "Model predictive control of regulation services from commercial buildings to the smart grid," in $A C C 2014$, June 2014, pp. 2226-2233.

[5] D. He, J. Mei, R. Harley, and T. Habeter, "Utilizing building-level demand response in frequency regulation of actual microgrids," in IECON, Nov 2013, pp. 2205-2210.

[6] M. Balandat, F. Oldewurtel, M. Chen, and C. Tomlin, "Contract design for frequency regulation by aggregations of commercial buildings," in 52nd Annual Allerton Conference on Communication, Control, and Computing, Sept 2014, pp. 38-45.

[7] H. Hao, B. Sanandaji, K. Poolla, and T. L. Vincent, "Potentials and economics of residential thermal loads providing regulation reserve," Energy Policy, vol. 79, pp. 115-126, Jan. 2015.

[8] V. Lakshmanan, M. Marinelli, A. M. Kosek, P. B. Nørgård, and H. W. Bindner, "Impact of thermostatically controlled loads' demand response activation on aggregated power: A field experiment," Energy, vol. 94, no. 1, pp. 705 - 714, 2016.

[9] J. Haack, B. Akyol, B. Carpenter, R. Pratt, and T. Carroll, "Volttron: An agent platform for integrating electric vehicles and smart grid," in ICCVE 2013, Las Vegas, NV 2013.

[10] E. Camacho and C. Bordons, "Model predictive control," Springer 2007. 